\title{
TNF $\alpha$-induced cardioprotection is independent of the activation of the prosurvival kinase Erk
}

\section{Sarin Somers, Aurelien Engerer and Sandrine Lecour}

Hatter Institute for Cardiology Research, Department of Medicine, Faculty of Health Sciences, University of Cape Town

\author{
Address for correspondence: \\ Dr Sandrine Lecour \\ Hatter Institute for Cardiology Research \\ Department of Medicine \\ Faculty of Health Sciences \\ University of Cape Town \\ Observatory \\ 7925 \\ South Africa
}

Email:

Sandrine.lecour@uct.ac.za

\section{INTRODUCTION}

Cellular protection of the heart is the new therapeutic challenge following successes achieved with reperfusion therapy. ${ }^{(1,2)}$ A powerful biological phenomenon that is being scientifically exploited to dissect out cell survival programs is known as ischaemic preconditioning. ${ }^{(3)}$ Ischaemic preconditioning is evoked by sublethal transient ischaemia that paradoxically results in increased tolerance against subsequent lethal ischaemia. Identification of cell survival programs activated by preconditioning may identify new therapeutic targets for future drug development to promote cell survival in the heart.

Tumour Necrosis Factor alpha (TNF $\alpha)$ is a pleiotropic cytokine that has been implicated to play a role in cellular inflammation growth, apoptosis but also cell survival.(4) We have previously reported that ischaemic preconditioning evokes an upregulation of cardiac steady-state TNF $\alpha$ peptide levels in the mouse heart and a loss of protection was associated with ischaemic preconditioning in TNF $\alpha$ deficient mice. ${ }^{(5)}$ In addition, we have demonstrated that TNF $\alpha$ could mimic ischaemic preconditioning in a dose and time-dependent manner in the rat heart. ${ }^{(6,7)}$

\section{ABSTRACT}

Introduction:Tumour Necrosis Factor alpha (TNF $\alpha$ ) contributes to cardiac dysfunction following ischaemia-reperfusion. Nonetheless, brief exposure to exogenous low doses of TNF $\alpha$ can mimic ischaemic preconditioning and thus be cardio-protective. The extracellular signal-regulated kinase (Erk) has been implicated in the protection against ischaemia-reperfusion and is known to be activated by TNF $\alpha$. However, whether Erk activation contributes to TNF $\alpha$-induced cardio-protection remains unknown.

Methods: PD 98059 (10 $\mu \mathrm{M})$, an Erk inhibitor, was used to evaluate the role of this prosurvival kinase with respect to infarct size (expressed as a percentage of the area at risk) in isolated rat hearts subjected to ischaemic preconditioning (IPC) or TNF $\alpha$ preconditioning (TNF $\alpha \mathrm{P})$. Western blot analyses were used to determine the degree of Erk phosphorylation after the application of the preconditioning stimulus.

Results: Pre-treatment of the hearts with $0.5 \mathrm{ng} / \mathrm{ml}$ of TNF $\alpha$ (for $7 \mathrm{~min}$ ) or 2 cycles of $5 \mathrm{~min}$ ischaemia-reperfusion prior to $30 \mathrm{~min}$ regional index ischaemia and $2 \mathrm{~h}$ of reperfusion reduced the infarct size by $76 \%$ and $88 \%$, respectively, versus control. Western blot analysis of isolated rat hearts revealed that IPC but not TNF $\alpha P$ activated phosphorylation of Erk ( $+54 \%$ for IPC vs. control). Coadministration of PD 98059 during the preconditioning stimulus did not influence the infarct size.

Conclusion: These findings confirm that activation of TNF $\alpha$ may be considered as a novel therapeutic approach against ischaemic heart disease and that its effect is independent of the activation of the classic prosurvival factor Erk. SAHeart 2009; 6:168-172

However, the signalling pathways involved in the cardioprotective effect of $\mathrm{TNF} \alpha$ remain poorly understood. Similar to ischaemic preconditioning, the protective effect of TNF $\alpha$ occurs via the sphingolipids, ${ }^{(6)}$ generation of free radicals, ${ }^{(6,8)}$ the activation of the signal transducer and activator of transcription-3 $(\text { STAT-3) })^{(9)}$ and the phosphorylation of $\mathrm{Bcl}-2$ antagonist of cell death (BAD). ${ }^{(9)}$ While ischaemic preconditioning has been shown to confer cardio-protection via the activation of the prosurvival kinase Akt, ${ }^{(10)}$ we were surprised to report that TNF $\alpha$ can 
protect independently of the activation of Akt. $(9,11)$ The necessity for the activation of the prosurvival kinase extracellular signalregulated kinase (Erk) during the ischaemic preconditioning stimulus has led to conflicting data in the literature. ${ }^{(12,13)}$ However, this prosurvival kinase has never been studied during a TNF $\alpha$ preconditioning stimulus.

Therefore, using an isolated rat heart model, the aim of this study is to investigate whether both ischaemic preconditioning- and $\mathrm{TNF} \alpha$ - induced cardio-protection require the activation of Erk during the preconditioning stimulus also called the trigger phase.

\section{METHODS}

Animal groups

All the experiments were conducted on adult male Long-Evans weighing 250-300g in accordance with the Guide for the Care and Use of Laboratory Animals (National Academic Press, Washington DC, 1996). The University of Cape Town Medical School Research Ethics Committee approved all experiments.

\section{Experimental protocols}

Rats were anesthetised with $60 \mathrm{mg} / \mathrm{kg}$ intraperitoneal sodium pentobarbitone and were given an intravenous injection of 200IU heparin. Hearts were excised rapidly and perfused retrogradely using the Langendorff technique at a constant pressure $\left(100 \mathrm{~cm} \mathrm{H}_{2} \mathrm{O}\right)$ with oxygenated Krebs-Henseleit buffer as previously described.(6) A balloon was inserted through the left atrium into the left ventricle and the left-ventricular end diastolic pressure (LVEDP) was adjusted between 4 and $8 \mathrm{mmHg}$. Cardiac parameters were monitored continuously and included heart rate (HR), left ventricular developed pressure (LVDP: difference between left ventricular end-systolic pressure and end-diastolic pressure) and the coronary flow (CF).

The perfusion protocol is shown in Figure I. All hearts were allowed to equilibrate for $15 \mathrm{~min}$ and were consequently subjected to a standard 30 min regional ischaemia (induced by tightening a snare around the left coronary artery) followed by $120 \mathrm{~min}$ of reperfusion. Ischaemic preconditioning (IPC) was performed by 2 cycles of $5 \mathrm{~min}$ global ischaemia-reperfusion prior to the standard ischaemia. TNF $\alpha(0.5 \mathrm{ng} / \mathrm{ml})$ was given for $7 \mathrm{~min}$ followed by a 10 min of wash out period before the standard ischaemia. Additional groups were perfused with the MAPK/Erk I/2 kinase

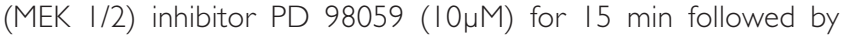
5 min washout prior to the ischaemia-reperfusion insult.

For infarct size measurement, the coronary artery was reoccluded at the end of the reperfusion period and a solution of 2.5\% Evans Blue was perfused to delineate the area at risk. Hearts were then frozen and cut into slices, incubated in sodium phosphate buffer containing $1 \% \mathrm{w} / \mathrm{v}$ triphenyltetrazolium chloride (TTC) for $15 \mathrm{~min}$ to visualise the unstained infarcted region. Infarct and risk zone areas were determined with planimetry and infarct was expressed as a percentage of the risk zone.

\section{Western blot analysis}

Hearts from control or preconditioned rat hearts were collected prior to the $30 \mathrm{~min}$ of regional ischaemia. The ventricular tissue was excised and freeze-clamped in liquid nitrogen before being stored at -80C. Cytosolic proteins were extracted as previously published. ${ }^{(9)}$ Phosphorylated proteins (phospho-Erk, thr 202/thr 204), total levels of Erk and B-actin were analysed by SDS-PAGE immune-electrophoresis using antibodies from Cell Signalling Technology. Equal loading was verified with Ponceau staining or with B-actin and levels of phosphorylated proteins were normalised to their total protein levels done in the same samples and in the same conditions but on a separate




membrane. Relative densitometry was determined using a computerised software package.

\section{Pharmacologic agents}

Recombinant rat tumour necrosis factor alpha was obtained from Pepro Tech EC Ltd (London, UK). All the chemicals were obtained from Sigma Chemical Company (St Louis, MO, USA).

\section{Statistical analysis}

Data are presented as mean \pm SEM $(n \geq 6)$. Comparisons between multiple groups were performed by one-way ANOVA followed by the Student-Newman-Keul post hoc test (Graph Pad Instat). A value of $p<0.05$ was considered statistically significant.

\section{RESULTS}

PD 98059 does not influence the infarct size in ischaemic or

\section{TNF $\alpha$ preconditioning.}

To investigate the role of Erk 1/2, we measured infarct size as the percentage of the area at risk in hearts perfused with an inhibitor of MEK I/2 (PD 98059). Ischaemic control hearts had an area at risk of $46.4 \pm 1.5 \%$ and an infarct size of $28.4 \pm 3.7 \%$ (Figure 2). The size of the infarct confirms previous data from

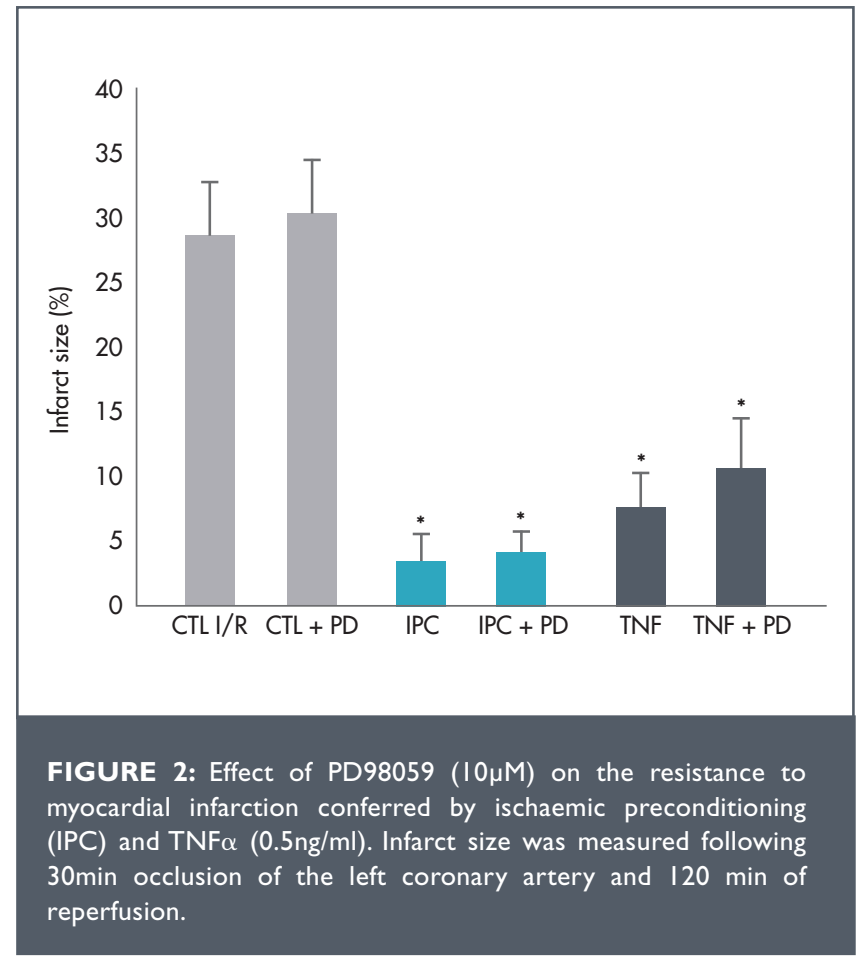

*p $<0.05$ versus control ischaemia/reperfusion group (CTL I/R).

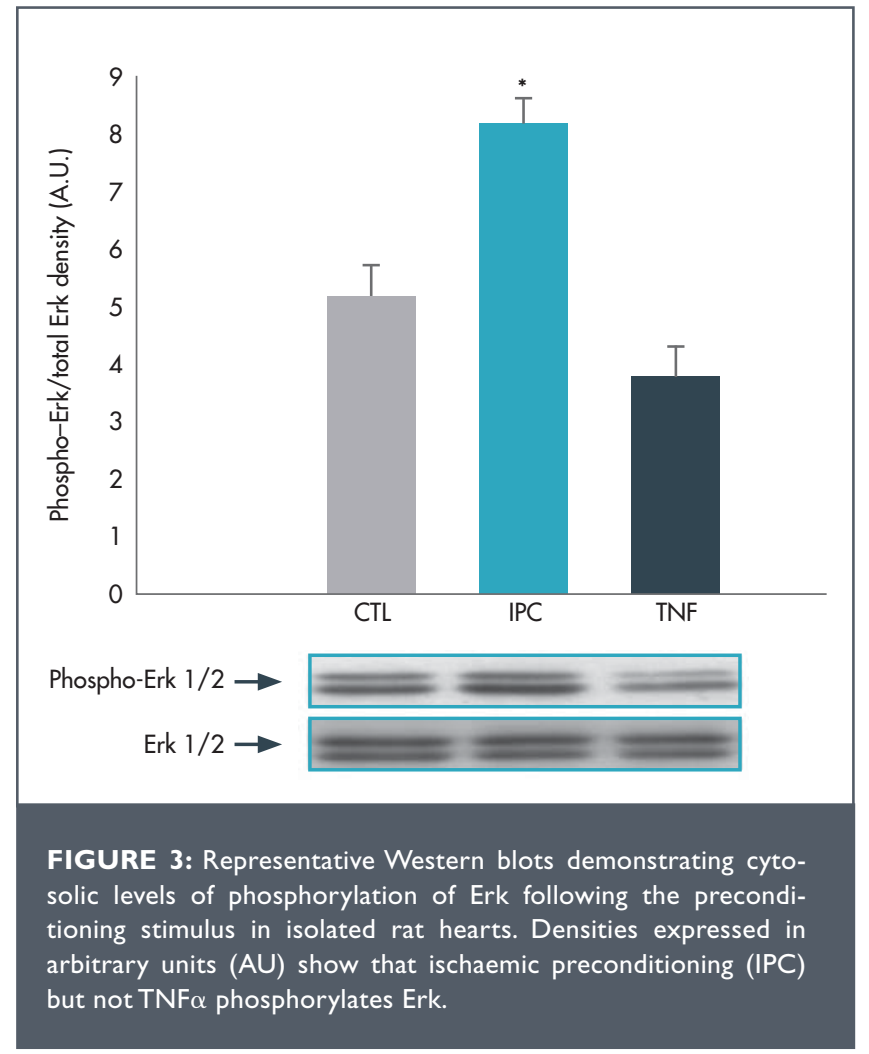

* $p<0.05$ versus control group (CTL).

our laboratory. ${ }^{(6,9,14)}$ Although the area at risk did not differ amongst the different groups (data not shown), both IPC and pharmacological preconditioning with TNF $\alpha$ reduced the infarct size compared with the ischaemic control group $(3.2 \pm 1.6$ and $6.6 \pm 2.0 \%$ respectively; $\mathrm{p}<0.001$ vs. ischaemic control group). When PD98059 was given during the preconditioning stimulus, the protective effects of neither IPC nor TNF $\alpha$ were abrogated (3.9 \pm 1.4 and $10.6 \pm 3.4 \%$ respectively; $p<0.001$ vs. ischaemic control group). Of note, PD98059, given on its own, did not affect the infarct size compared with the ischaemic control group $(30.5 \pm 4.0 \%$, ns vs. ischaemic control group).

IPC but not TNF $\alpha$ activates phosphorylation of Erk I/2 during the preconditioning stimulus.

We explored whether Erk was phosphorylated following the preconditioning stimulus with ischaemia or with TNF $\alpha$. As shown in Figure 3, IPC significantly phosphorylated cytosolic Erk I/2 compared with the control group $(8.1 \pm 0.4$ arbitrary units (A.U.) for IPC versus $5.1 \pm 0.5$ for the control group, $p<0.0$ I). In contrast, TNF $\alpha$ preconditioning stimulus did not affect phosphorylation of Erk I/2 (3.7 \pm 0.5 A.U.). 


\section{DISCUSSION}

Using the isolated rat heart model, our novel data show - for the first time - that TNF $\alpha$ mimics ischaemic preconditioning independently of the activation of the prosurvival kinase Erk 1/2 prior to the ischaemic insult. In addition, we report that ischaemic preconditioning is associated with an increased phosphorylation of Erk 1/2 prior to the index ischaemia but its inhibition does not abrogate the protection afforded by ischaemic preconditioning.

Previous studies have explored the role of Erk in ischaemic preconditioning but the results have led to conflicting data. Using an in vivo model of ischaemia/reperfusion in rats, Fryer et al. reported an increased phosphorylation of Erk following a preconditioning stimulus with either ischaemia or an opioid receptor agonist. ${ }^{(15)}$ In addition, inhibition with PD 98059 abolished the cardio-protective effect of both preconditioning stimuli. Similarly, inhibition of Erk with PD 98059 could abolish the ischaemic preconditioning effect in the porcine heart in vivo.(16) In contrast, Behrends et al. failed to find any correlation between Erk phosphorylation and the protection afforded by ischaemic preconditioning.(17) In the isolated rat heart model, Mocanu et al. demonstrated that Erk phosphorylation occurred as a result of ischaemic preconditioning but was not required for protection. (13) Using different doses of PD 98059 to inhibit the phosphorylation of Erk they also confirm that their findings were not dependent on the concentration of the inhibitor used in their model. Using the same model with a different strain of rats, our data correlated with Mocanu's findings, thus suggesting that the discrepancies described in the literature may be related to the different species and models used.

In ischaemic preconditioning ${ }^{(18)}$ and with numerous pharmacological agents ${ }^{(19-21)}$ the phosphorylation of this kinase is required at the onset of reperfusion as part of the activation of the Reperfusion Injury Salvage Kinase pathway (RISK). However, we were surprised to report that pharmacological preconditioning with TNF $\alpha$ protects independently of the phosphorylation of Erk at the time of reperfusion. ${ }^{(9)}$ In addition, its inhibition during the reperfusion phase did not alter the protection afforded by the TNF $\alpha$ stimulus. (9) If we had previously shown that Erk is not a mediator in TNF $\alpha$-induced preconditioning our present data strongly suggest that Erk does not act as a trigger either. In cardiomyocytes, previous reports have described a phosphorylation of Erk following TNF $\alpha$ exposure but the cells were exposed to higher and harmful doses of TNF $\alpha .^{(22,23)}$ In addition, the activation of Erk in the cardiomyocytes exposed to $10 \mathrm{ng} / \mathrm{ml}$ of TNF $\alpha$ (dose 20 times higher than the dose used in our model) was so low that it was undetectable with a classic Western blot technique. ${ }^{(23)}$ It is appropriate to suggest that a very small phosphorylation of Erk may have occurred in our model but its inhibition with PD 98059 did not alter the cardio-protective effect of TNF $\alpha$, therefore suggesting that Erk is not required for TNF $\alpha$ to promote cardio-protection. However, it is important to acknowledge that the use of non specific pharmacological inhibitors such as PD 98059 is a limitation to our study. Unfortunately, there is no inhibitor specifically targeting the kinase Erk and further studies, using either genetically modified mice such as Erk null mice or siRNA techniques, should be considered to confirm our findings.

The signalling cascade leading to cardio-protection with TNF $\alpha$ still remains poorly understood. Tanno et al. reported that TNF $\alpha$ induced protection of the murine heart is independent of p38-MAPK activation. (24) We have shown that the activation of sphingolipids, protein kinase $C$, the mitochondrial potassium ATP-dependent and reactive oxygen species were required during the preconditioning phase for TNF $\alpha$ to confer cardioprotection. ${ }^{(6,8)}$ The mitochondrial permeability transition pore and the calcium-activated potassium channel have also been suggested.(25) Most importantly, we have recently demonstrated that the activation of STAT-3, as part of the Survivor Activating Factor Enhancement (SAFE) pathway, is a novel and alternative protective pathway that is activated during the trigger phase in both ischaemic preconditioning and pharmacological preconditioning with TNF $\alpha{ }^{(26,27)}$

In conclusion, our present data demonstrate that both ischaemic preconditioning and TNF $\alpha$ can confer protection against ischaemiareperfusion, independently of the activation of the prosurvival kinase Erk prior to ischaemia. Combined with our already published work, these data add proof that multiple and independent protective paths can be activated within the heart and that these pathways do not need to be all activated to protect the cells from dying. However, additional work is needed to understand 
whether activation of several pathways at the same time can confer an additive effect to maximise the protection or if the threshold of protection is already achieved by activation of a single path.

\section{ACKNOWLEDGMENTS}

This work was supported in part by the Circulatory Disorders Research Fund, a grant from the National Research Foundation and the Inter-university Cape Heart Group of the South African Medical Research Council. S.L was supported by a Medical Research Council Career Award and a Servier Senior Fellowship.

\section{REFERENCES}

I. Yellon DM, Hausenloy DJ. Myocardial reperfusion injury. $N$ Engl J Med 2007;357: I 1 21-35.

2. Theroux P. Myocardial cell protection: a challenging time for action and a challenging time for clinical research. Circulation 2000; 10 1:2874-6.

3. Murry CE, Jennings RB, Reimer KA. Preconditioning with ischaemia: a delay of lethal cell injury in ischaemic myocardium. Circulation 1986;74:1 I 24-36.

4. Mann DL. Stress-activated cytokines and the heart: from adaptation to maladaptation. Annu Rev Physiol 2003;65:81- 101.

5. Smith RM, Suleman N, McCarthy J, et al. Classic ischaemic but not pharmacologic preconditioning is abrogated following genetic ablation of the TNF $\alpha$ gene. Cardiovasc Res 2002;55:553-560.

6. Lecour S, Smith RM, Woodward B, et al. Identification of a novel role for sphingolipid signaling in TNF alpha and ischaemic preconditioning mediated cardioprotection. J Mol Cell Cardiol 2002;34:509-18.

7. Deuchar GA, Smith R, Lecour S, et al. In vivo cardioprotective role of TNFalpha. J Mol Cell Cardiol 2005;38: I 0 I 6. Abstract.

8. Lacerda L, Smith RM, Opie L, et al. TNFalpha-induced cytoprotection requires the production of free radicals within mitochondria in $\mathrm{C}(2) \mathrm{C}(12)$ myotubes. Life Sci 2006.

9. Lecour S, Suleman N, Deuchar GA, et al. Pharmacological preconditioning with tumor necrosis factor-alpha activates signal transducer and activator of transcription-3 at reperfusion without involving classic prosurvival kinases (Akt and extracellular signal-regulated kinase). Circulation 2005; | | 2:39 | I-8.

10. Tong $H$, Chen $W$, Steenbergen $C$, et al. Ischaemic preconditioning activates phosphatidylinositol-3-kinase upstream of protein kinase C. Circ Res 2000;87: 309-15

11. Suleman N, Somers S, Smith R, et al. Dual activation of STAT-3 and Akt is required during the trigger phase of ischaemic preconditioning. Cardiovasc Res 2008;79:127-33.

12. Fryer RM, Hsu AK, Eells JT, et al. Opioid-induced second window of cardioprotection: potential role of mitochondrial KATP channels. Circ Res 1999;84: 846-51.

13. Mocanu MM, Bell RM, Yellon DM. PI3 kinase and not p42/p44 appears to be implicated in the protection conferred by ischaemic preconditioning. J Mol Cell Cardiol 2002;34:661-8.
14. Lecour S, Rochette L, Opie L. Free radicals trigger TNFalpha-induced cardioprotection. Cardiovasc Res 2005;65:239-43

15. Fryer RM, Pratt PF, Hsu AK, et al. Differential activation of extracellular signa regulated kinase isoforms in preconditioning and opioid-induced cardioprotection. J Pharmacol Exp Ther 2001;296:642-9.

16. Strohm C, Barancik T, Bruhl ML, et al. Inhibition of the ER-kinase cascade by PD98059 and UOI26 counteracts ischaemic preconditioning in pig myocardium. J Cardiovasc Pharmacol 2000;36:218-29.

17. Behrends M, Schulz R, Post $H$, et al. Inconsistent relation of MAPK activation to infarct size reduction by ischaemic preconditioning in pigs. Am J Physiol Heart Circ Physiol 2000;279:HIIII-9.

18. Hausenloy DJ, Mocanu MM, Yellon DM. Cross-talk between the survival kinases during early reperfusion: its contribution to ischaemic preconditioning. Cardiovasc Res 2004;63:305-12.

19. Gross ER, Hsu AK, Gross GJ. Opioid-induced cardioprotection occurs via glycogen synthase kinase beta inhibition during reperfusion in intact rat hearts. Circ Res 2004;94:960-6

20. Yang $X M$, Krieg $T$, Cui $L$, et al. NECA and bradykinin at reperfusion reduce infarction in rabbit hearts by signaling through PI3K, ERK, and NO. J Mol Cell Cardiol 2004;36:41।-21.

21. Philipp S, Critz SD, Cui L, et al. Localising extracellular signal-regulated kinase (ERK) in pharmacological preconditioning's trigger pathway. Basic Res Cardiol 2006; 101:159-67.

22. Tantini B, Pignatti C, Fattori M, et al. NF-kappaB and ERK cooperate to stimulate DNA synthesis by inducing ornithine decarboxylase and nitric oxide synthase in cardiomyocytes treated with TNF and LPS. FEBS Lett 2002;5 12:75-9.

23. Clerk A, Harrison JG, Long CS, et al. Pro-inflammatory cytokines stimulate mitogen-activated protein kinase subfamilies, increase phosphorylation of c-Jun and ATF2 and upregulate c-Jun protein in neonatal rat ventricular myocytes. J Mol Cell Cardiol 1999;31:2087-99.

24. Tanno M, Gorog DA, Bellahcene M, et al. Tumor necrosis factor-induced protection of the murine heart is independent of p38-MAPK activation. J Mol Cell Cardiol 2003:35: I523-7.

25. Gao Q, Zhang SZ, Cao CM, et al.The mitochondrial permeability transition pore and the $\mathrm{Ca} 2+$-activated $\mathrm{K}+$ channel contribute to the cardioprotection conferred by tumor necrosis factor-alpha. Cytokine 2005;32:199-205.

26. Lecour S. Activation of the protective Survivor Activating Factor Enhancement (SAFE) pathway against reperfusion injury: Does it go beyond the RISK pathway? J Mol Cell Cardiol 2009; 47:32-40.

27. Lecour S. Multiple protective pathways against reperfusion injury: A SAFE path without Aktion? J Mol Cell Cardiol 2009;46:607-9. 